\title{
Does Correlated Firing Underlie Attention Deployment in Frontal Cortex?
}

\author{
Moein Esghaei ${ }^{1,2 *}$ and Cheng Xue ${ }^{2 *}$ \\ ${ }^{1}$ Cognitive Neurobiology Laboratory, School of Cognitive Sciences, Institute for Research in Fundamental Sciences (IPM), Tehran 19395, Iran, and \\ ${ }^{2}$ Cognitive Neuroscience Laboratory, German Primate Center (DPZ), Goettingen 37077, Germany \\ Review of Oemisch et al.
}

Attention is an essential cognitive ability that animals and humans rely on to survive. It allows complex nervous systems to selectively process the most behaviorally relevant sensory information. While an abundance of literature has demonstrated attentional modulation of neuronal activity in sensory areas (Treue, 2001; Maunsell and Treue, 2006), it is much less clear which brain areas control this modulation (Petersen and Posner, 2012). One candidate region is the prefrontal cortex (PFC), which makes reciprocal projections with almost all sensory and motor cortical areas, as well as many subcortical structures (Miller and Cohen, 2001). Studies on the cross-areal interaction between prefrontal and sensory areas have identified PFC as a major control center for directing attention to a location (Moore and Armstrong, 2003; Gregoriou et al., 2009), a feature, or an object (Zaksas and Pasternak, 2006; Baldauf and Desimone, 2014). An additional candidate is anterior cingulate cortex (ACC), which has a close functional connectivity with PFC (Womelsdorf et al., 2014). Although numerous studies have

Received Nov. 23, 2015; revised Dec. 25, 2015; accepted Dec. 30, 2015.

This work was supported by the Iranian Cognitive Sciences and Technologies Council. We thank Stefan Treue and Mohammad Reza Daliri for their kind comments on an earlier version of the article.

The authors declare no competing financial interests.

*M.E. and C.X. contributed equally to this work.

Correspondence should be addressed to Moein Esghaei, Cognitive Neuroscience Laboratory, German Primate Center (DPZ), Kellnerweg 4, Goettingen 37077, Germany. E-mail: esghaei@ipm.ir.

DOI:10.1523/JNEUROSCI.4211-15.2016

Copyright $\odot 2016$ the authors $\quad 0270-6474 / 16 / 361791-03 \$ 15.00 / 0$ probed the role of these two frontal areas in controlling attention, it is not clear how attention signals are integrated within and between these regions.

In a recent paper, Oemisch et al. (2015) studied correlations between instantaneous firing rates of neurons located in different frontal cortex substructures in an attention task. They trained two rhesus monkeys to maintain their gaze on a central fixation point on a computer screen while two peripheral drifting gratings, each with a different color, were presented to the left and right visual hemifield. After the color of the fixation point changed to that of either of the gratings, the monkeys had to covertly attend to the corresponding grating (the target) without diverting their gaze from the fixation point (for experimental details, see Fig. 1). The authors recorded single and multiunit activities from dorsal and lateral PFC, ventromedial PFC, and ACC while the monkeys performed the task.

Oemisch and colleagues (2015) report three major findings. First, starting 280 ms after the monkeys were cued to the target location, the instantaneous activities of those pairs of neurons that were recorded simultaneously became correlated across trials. These correlations were especially pronounced for crossareal neuron pairs recorded from ACC and PFC, suggesting that a neural interaction occurs between these two areas. This correlation does not appear to be a simple side effect of engaging spatial attention, but rather appears to reflect where the monkeys attend, because a significant portion of neuron pairs from ACC and PFC showed distinct correlations when the monkeys attended contralaterally rather than ipsilaterally to the recorded hemisphere (Oemisch et al., 2015, their Fig. 5Biii). Second, the activity of dorsal PFC (dPFC) neurons at a given time bin between 0 and $800 \mathrm{~ms}$ after cue onset was correlated with the activity of ACC neurons in subsequent time bins, suggesting that $\mathrm{dPFC}$ neurons play a role in driving ACC neurons. Third, the ACC-PFC correlation was statistically significant only when spike trains were smoothed using Gaussian kernels with widths of 50-200 ms. Since this time-scale is far larger than that of monosynaptic communication, it suggests that attention control involves coordination of large-scale brain networks.

To quantify interneuronal interactions, Oemisch and colleagues (2015) calculated correlations between the instantaneous activities of neuron pairs. This measure quantifies, for a given instant from cue onset, the correlation of spike rates for a given neuron pair across trials. However, their behavioral paradigm might create spurious intertrial correlations: given that the response of a considerable proportion of neurons in the frontal cortex depends on the visual properties of behaviorally relevant stimuli (Zaksas and Pasternak, 2006; Bichot et al., 2015), the response of these neurons will change when attention is switched between the left and right visual hemifield 


\section{Stimulus rotation}

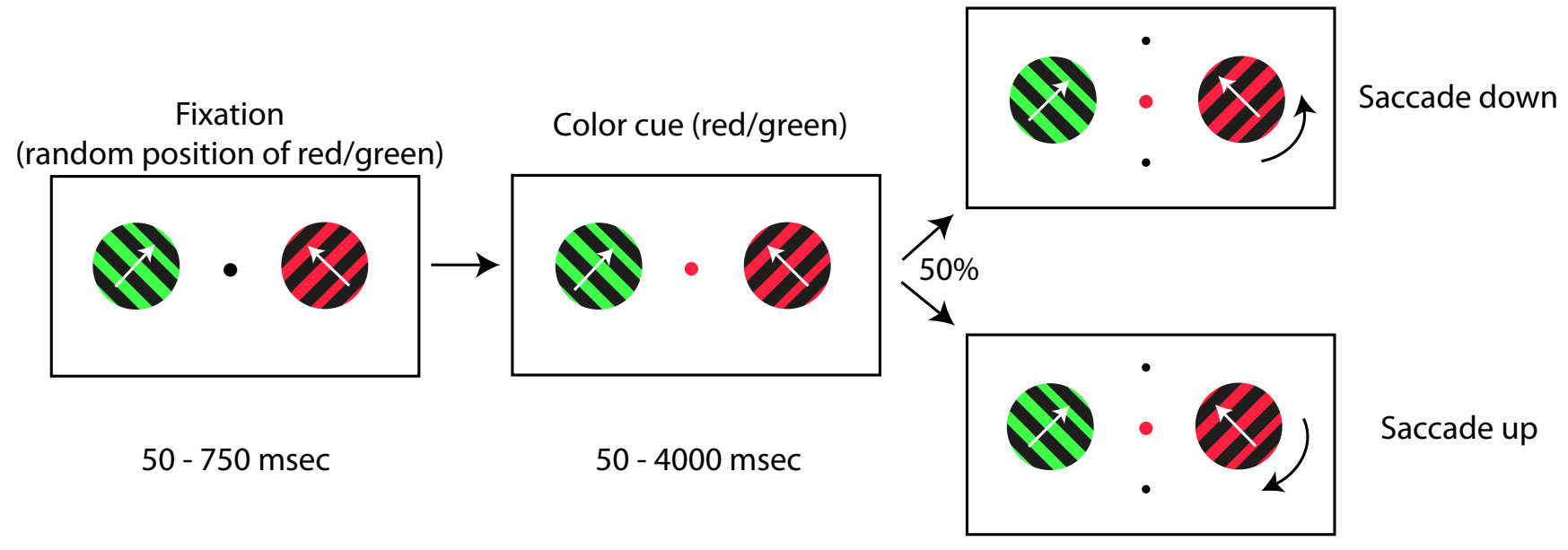

Figure 1. Behavioral task used by 0emisch et al. (2015). Monkeys had to maintain their gaze on a central point while a $+45^{\circ}$ and a $-45^{\circ}$ drifting grating were presented to the left and right visual hemifield, respectively. In each trial, one of the gratings was randomly selected to be green and the other, red. At a random time between 50 and 750 ms, the fixation point changed color to red or green, cueing the monkeys to covertly shift their attention to the matching (target) grating. After a random period of $50-4000$ ms, one of the gratings underwent a clockwise or counterclockwise change in direction of motion. Monkeys were trained to make an upward or downward saccade if the target grating underwent a clockwise or counterclockwise change in direction, respectively. The white arrows represent drifting direction and were not shown in the actual experiment (details originally described by Kaping et al., 2011).

(to gratings drifting at $+45^{\circ}$ and $-45^{\circ}$, respectively). This alone can explain the trial-by-trial correlation induced after cue onset when the visual information about the attended stimulus has reached the neurons: assuming a pair of neurons that are both selective to the contralateral grating (which plays a major role in the main finding of the paper; Oemisch et al., 2015, their Fig. $5 A$ ), both will increase their firing rate in trials on which the monkeys attend contralaterally, and decrease their firing rate when the animals attend ipsilaterally, thus creating an intertrial correlation. The influence of such an effect can be avoided by only analyzing trials in which the monkeys attend to the same drifting direction. Similarly, when the authors compare the interneuronal correlation between contralateral and ipsilateral attention shifts (Oemisch et al., 2015, their Fig. 5Biii), any difference can be accounted for by the difference in signal-tonoise ratio of the neural response to the preferred versus nonpreferred stimulus. This could be controlled by computing the correlations for each neuron, using a subset of trials in which the neuron does not show a significant change in spike rate between contralateral/ipsilateral attention shifts. Alternatively, future experiments could present two identical stimuli in the two visual hemifields to induce the same neural responses between contralateral/ipsilateral attention shifts. Whether the interneuronal correlation will remain statistically significant after removing the component induced by differential visual stimulations is an important concern, however, given the small correlation values (average Pearson correlation coefficient $<0.02$ ) (Oemisch et al., 2015, their Fig. $2 B$ ) compared with similar studies in sensory areas (Mitchell et al., 2009).

To investigate the role of attentionevoked neural correlation between ACC and dPFC neurons, Oemisch et al. (2015) quantified the directionality of the interneuronal interaction using a directionality index, proposed earlier (Paz et al., 2007). This index measures, for a given pair of neurons (A and B), the difference between the mean trial-by-trial correlation of instantaneous neural activities across time-pairs with the activity of neuron A proceeding neuron $\mathrm{B}$ and those time-pairs with the activity of neuron $\mathrm{B}$ proceeding neuron $\mathrm{A}$. This gives a measure of whether either of the neurons in a given pair activated the other neuron. However, this method suffers from dependence on the time scale it is applied to. As the authors point out, when changing the analysis period to $300-600 \mathrm{~ms}$ after cue onset (rather than $0-800 \mathrm{~ms}$ ), the directionality effect disappears unless the spike trains are smoothed by convolving an extra-large Gaussian kernel with the width of $400 \mathrm{~ms}$ rather than 50-200 ms. Therefore, to study the directionality of interneuronal interactions in a given interval, all constituent time windows should be investigated.

Oemisch et al. (2015) found that interneuronal correlations only emerged when the Gaussian kernel width used for smoothing spike trains was larger than 50 ms (Oemisch et al., 2015, their Figs. 1C,
$2 A, 3 C)$, with an understandable exception of $400 \mathrm{~ms}$ kernel width because it expanded beyond the analysis window (Oemisch et al., 2015, their Figs. 1D, 2E, $3 C)$. The authors interpret this as a characteristically slow time scale for coordination across areas. Yet the underlying mechanism could easily be further explored. The most straightforward interpretation of the effect of kernel width on firing correlation is that attention induced a certain pattern of neuronal events, which by themselves have stochastic time intervals that distribute $\sim 50 \mathrm{~ms}$ or longer; therefore, a consistent correlation across areas becomes evident only when a time window larger than $50 \mathrm{~ms}$ is used to overcome the stochasticity. Meanwhile, another study on the same dataset (Womelsdorf et al., 2014) demonstrated that there is an increase in the proportion of neuronal bursts (clusters of spikes intercepted with variable quiescent periods) after the attention cue onset. They further found that these bursts are synchronized with the narrow beta band $(12-20 \mathrm{~Hz})$ of local field potentials (LFP), which, probably not coincidentally, corresponds to a period of $>50 \mathrm{~ms}$. Therefore, we speculate that the characteristic slow time scale reflects the stochasticity between the bursts, which occurs within a period of beta-band rhythm. This hypothesis can be directly tested by looking into potential correlations between attentional effects on burstiness and interneuronal correlation. The authors made an attempt along this line when they reported no across-cell-pair correlation between burst 
proportions and correlation strengths. However, this lack of correlation is probably not surprising, given that different cell types in prefrontal cortex have distinct burst properties to start with, independent of their correlation induced by attention (shown on the same dataset by Ardid et al., 2015). Therefore, a more relevant test would address the link between the two effects within cell pairs, i.e., whether for a given neuron pair, the attentional modulation of neural correlation is stronger when calculated for bursty trials compared with less bursty trials. If there is no link between interneuronal correlation and burstiness, interneuronal correlation could be attributed an independent role in controlling attention.

In summary, Oemisch et al. (2015) provided evidence suggesting the involvement of interneuronal interaction between areas ACC and APFC when monkeys switched their attention to different entities. They also suggested that dPFC neurons control the activity of ACC neurons when deploying attention. However, there are at least three more steps to accomplish before reaching a firm conclusion about the role of interneuronal correlations in controlling attention: first, to disentangle the interneuronal correlation from differential neuronal responses evoked by stimulus variants; second, to in- vestigate all constituent time windows of postcue period for any potential role of $\mathrm{dPFC}$ in governing ACC for attention control; and third, to clarify the relationship between attentional modulations of correlated firing and burstiness-LFP synchronization.

\section{References}

Ardid S, Vinck M, Kaping D, Marquez S, Everling S, Womelsdorf T (2015) Mapping of functionally characterized cell classes onto canonical circuit operations in primate prefrontal cortex. J Neurosci 35:2975-2991. CrossRef Medline

Baldauf D, Desimone R (2014) Neural mechanisms of object-based attention. Science 344: 424-427. CrossRef Medline

Bichot NP, Heard MT, DeGennaro EM, Desimone R (2015) A source for feature-based attention in the prefrontal cortex. Neuron 88: 832-844. CrossRef Medline

Gregoriou GG, Gotts SJ, Zhou H, Desimone R (2009) High-frequency, long-range coupling between prefrontal and visual cortex during attention. Science 324:1207-1210. CrossRef Medline

Kaping D, Vinck M, Hutchison RM, Everling S, Womelsdorf T (2011) Specific contributions of ventromedial, anterior cingulate, and lateral prefrontal cortex for attentional selection and stimulus valuation. PLoS Biol 9:e1001224. CrossRef Medline

Maunsell JH, Treue S (2006) Feature-based attention in visual cortex. Trends Neurosci 29: 317-322. CrossRef Medline

Miller EK, Cohen JD (2001) An integrative the- ory of prefrontal cortex function. Annu Rev Neurosci 24:167-202. CrossRef Medline

Mitchell JF, Sundberg KA, Reynolds JH (2009) Spatial attention decorrelates intrinsic activity fluctuations in macaque area V4. Neuron 63 : 879-888. CrossRef Medline

Moore T, Armstrong KM (2003) Selective gating of visual signals by microstimulation of frontal cortex. Nature 421:370-373. CrossRef Medline

Oemisch M, Westendorff S, Everling S, Womelsdorf T (2015) Interareal spike-train correlations of anterior cingulate and dorsal prefrontal cortex during attention shifts. J Neurosci 35:13076-13089. CrossRef Medline

Paz R, Bauer EP, Paré D (2007) Learning-related facilitation of rhinal interactions by medial prefrontal inputs. J Neurosci 27:6542-6551. CrossRef Medline

Petersen SE, Posner MI (2012) The attention system of the human brain: 20 years after. Annu Rev Neurosci 35:73-89. CrossRef Medline

Treue S (2001) Neural correlates of attention in primate visual cortex. Trends Neurosci 24: 295-300. CrossRef Medline

Womelsdorf T, Ardid S, Everling S, Valiante TA (2014) Burst firing synchronizes prefrontal and anterior cingulate cortex during attentional control. Curr Biol 24:2613-2621. CrossRef Medline

Zaksas D, Pasternak T (2006) Directional signals in the prefrontal cortex and in area MT during a working memory for visual motion task. J Neurosci 26:11726-11742. CrossRef Medline 